\title{
Precise local blur estimation based on the first-order derivative
}

\author{
Henri Bouma *, Judith Dijk and Adam W.M. van Eekeren; \\ TNO, PO Box 96864, 2509 JG The Hague, The Netherlands.
}

\begin{abstract}
Blur estimation is an important technique for super resolution, image restoration, turbulence mitigation, deblurring and autofocus. Low-cost methods have been proposed for blur estimation. However, they can have large stochastic errors when computed close to the edge location and biased estimates at other locations. In this paper, we define an efficient, accurate and precise estimate that can be computed at the edge location based on the first-order derivative. Our method is compared and benchmarked against previous state-of-the-art. The results show that the proposed method is fast, unbiased and with low stochastic error.
\end{abstract}

Keywords: Blur estimation, PSF, autofocus, image restoration, image enhancement, turbulence, super resolution.

\section{INTRODUCTION}

Blur estimation is a technique that can be used for many practical applications, such as super resolution [10], image restoration, turbulence mitigation [8][9], quantification [3], deblurring and autofocus [16]. Many algorithms have been proposed to perform blur estimation in images and video. In a short survey, we show that some are computationally inefficient, and some require higher-order polynomials. Some seem very efficient and robust, but have large stochastic errors when computed close to the edge location and systematic errors at other locations, as will be shown and analyzed.

In this paper, we present an efficient, accurate and precise blur estimator that can be computed at the edge location. Furthermore, it is robust against small variations due to dislocation or noise. The novel estimator is based on the firstorder derivative. Our method is compared and benchmarked against previous state-of-the-art methods under different levels of blur, dislocations and noise to gain insight in the robustness. The capabilities and limitations of both state-ofthe-art and our methods are analyzed and described. The experimental results show that the presented method is fast, unbiased and with low stochastic error.

*henri.bouma@tno.nl; phone +31 88866 4054; http://www.tno.nl

Henri Bouma, Judith Dijk and Adam W. M. van Eekeren, "Precise local blur estimation based on the first-order derivative", Proc. SPIE, Vol. 8399, 839904 (2012); http://dx.doi.org/10.1117/12.918600

Copyright 2012 Society of Photo-Optical Instrumentation Engineers (SPIE). One print or electronic copy may be made for personal use only. Systematic reproduction and distribution, duplication of any material in this paper for a fee or for commercial purposes, or modification of the content of the paper are prohibited. 


\section{OVERVIEW OF EXISTING METHODS}

\subsection{Assumptions and notation}

We assume that an image contains blur that locally can be modeled as a convolution with an isotropic Gaussian pointspread function (PSF). Furthermore, we assume that the image contains at least a few regions where the luminance distribution is a straight step-edge that does not interfere with other patterns. The blur will be estimated locally at these step edges, and the blur is not required to be uniform over the whole image.

In this paper, we will use the following notations. The blurred step edge in image $S_{o}$ is parameterized by the under- and upper bound $\mathrm{U}_{1}$ and $\mathrm{U}_{2}$ respectively, step-height $\mathrm{H}\left(\mathrm{H}=\mathrm{U}_{2}-\mathrm{U}_{1}\right)$ and the Gaussian blur with standard deviation $\sigma_{0}$. Reblurring the image with Gaussian blur kernels $\sigma_{\mathrm{a}}$ and $\sigma_{\mathrm{b}}$ (where $\sigma_{\mathrm{a}}<\sigma_{\mathrm{b}}$ ) will generate the reblurred images $S_{\mathrm{a}}(\mathrm{x})$ and $\mathrm{S}_{\mathrm{b}}(\mathrm{x})$, with a total blurring of $\sigma_{\mathrm{ta}}=\sqrt{ }\left(\sigma_{\mathrm{o}}{ }^{2}+\sigma_{\mathrm{a}}{ }^{2}\right)$ and $\sigma_{\mathrm{tb}}=\sqrt{ }\left(\sigma_{\mathrm{o}}{ }^{2}+\sigma_{\mathrm{b}}{ }^{2}\right)$ respectively. The first- and second-order Gaussian derivatives [4] in the $\mathrm{x}$-direction will be denoted as $\mathrm{L}_{\mathrm{x}}$ and $\mathrm{L}_{\mathrm{xx}}$, in y-direction as $\mathrm{L}_{\mathrm{y}}$ and $\mathrm{L}_{\mathrm{yy}}$ and in the gradient direction $\mathrm{L}_{\mathrm{w}}$ and $\mathrm{L}_{\mathrm{ww}}$. Most of the equations below are applied exactly at the center of the step-edge, unless specified differently.

\subsection{Overview of existing methods}

In this subsection, we will discuss five types of methods for blur estimation.

1) Direct model fit

The first type uses a direct fit of a model for the point-spread function (PSF) or the edge-spread function (ESF). For example, Pham [15] fits an error-function (erf) model to the edge profile that contains pairs of edge location-offsets and edge-intensities.

\section{2) Third-order polynomials}

The second type is based on third-order polynomials. Kayargadde [14] emphasizes that the signal-to-noise ratio decreases for higher order polynomials. Therefore, they tried to use coefficients of the lowest order possible. In their approach, they need at least coefficients up to order three to solve the edge parameters.

$$
\sigma_{o}=\sigma_{a}\left[\left(\frac{2 H_{w w}{ }^{2}}{H_{w}{ }^{2}}-\frac{\sqrt{6} H_{w w w}}{H_{w}}\right)^{-1}-1\right]^{1 / 2}
$$

Inspired by the work of Kayargadde, we can make a similar derivation for Gaussian derivatives instead of their Hermite coefficients. In this case the equation becomes:

$$
\sigma_{t a}=\sqrt{-\frac{L_{w}^{2}}{L_{w} L_{w w w^{-}} L_{w w}{ }^{2}}}
$$

Eq. 2 can also be applied close to the edge location. When applied exactly at the center of the edge $\mathrm{L}_{\mathrm{ww}}$ is 0 , so at the edge location the equation reduces to:

$$
\sigma_{t a}=\sqrt{-\frac{L_{w}}{L_{w w w}}}
$$

Chiang e.a. [6] also proposed to use a cubic polynomial:

$$
\sigma_{t a}=\sqrt{\frac{r+\sqrt{s}}{2}} ; r=-\frac{Q^{\prime}(x)}{Q^{\prime \prime \prime}(x)} ; s=r^{2}-4 r\left(x-x_{0}\right)^{2}
$$

at the edge location $\left(\mathrm{x}=\mathrm{x}_{0}\right)$ it reduces to the same solution as Eq. 3 .

3) Distance to zero-crossings of third-order derivative

The third type of methods for blur estimation is based on the distance between the zero-crossings of the third-order derivative. Elder and Zucker [11] estimate the amount of blur by measuring the distance $\mathrm{d}_{3}$ between the two zerocrossing of the third derivative $\left(\mathrm{L}_{\mathrm{www}}\right)$ :

$$
\sigma_{t a}=d_{3} / 2
$$


Bae and Durand [1] proposed an improvement of Elder and Zucker [11]. They found that the localization of the zerocrossings of the third derivative is not robust. Therefore, instead of measuring the distance between the actual zerocrossings, they used a brute-force strategy to fit multiple models of the second derivative at different scales to the pixel responses and find the distance with the least square fitting error.

4) First-order derivative and step height

The fourth type of methods for blur estimation is based on the gradient magnitude and the step height. Zhang [17] proposed a least-squares fit for solving $\mathrm{H}$ and $\sigma_{\mathrm{o}}$, based on an equation that uses a first-order derivative $\mathrm{L}_{\mathrm{w}}$ at multiple scales connected by edge focussing:

$$
L_{w} \sqrt{2 \pi} \sigma_{a}=\frac{H \sigma_{a}}{\sqrt{\sigma_{o}^{2}+\sigma_{a}^{2}}}
$$

Dijk et al. [7] proposed to use a similar relation:

$$
M_{w}=\frac{H \sigma_{a}}{\sigma_{t a} \sqrt{2 \pi}}
$$

In this equation we see that the derivative $\left(\mathrm{L}_{\mathrm{w}}\right)$ was replaced by a the normalized derivative $\left(\mathrm{M}_{\mathrm{w}}\right)$ and therefore the term ' $\sigma_{\mathrm{a}}$ ' was added in the equation. The response $\mathrm{M}_{\mathrm{w}}$ was computed for multiple scales $\left(\sigma_{\mathrm{a}}\right)$ and the measured responses were fitted to the theoretical responses. The minimization fit was done by the Levenberg-Marquardt method using 8 different scales.

Of course, the equation of [17] can easily be simplified by removing $\sigma_{\mathrm{a}}$ at both sides. Furthermore, the edge height $\mathrm{H}$ can be calculated directly based on the average intensity on each side of the edge (excluding a transition range). Recently, Cao [5] published this simplification that allows direct computation of the amount of blur without a minimization or fitting procedure:

$$
\sigma_{t a}=\frac{H}{L_{w} \sqrt{2 \pi}}
$$

5) Zeroth-order blur at two scales

The fifth type of blur estimation uses (zeroth-order derivative) Gaussian blurring kernels at different scales. Hu and Haan [12][13] proposed an approximation based on two blurring kernels. They showed clearly better results than Elder and Zucker [11].

$$
\sigma_{o} \approx \frac{\sigma_{a} \sigma_{b}}{\left(\sigma_{b}-\sigma_{a}\right) R+\sigma_{b}} ; R=\frac{S_{o}-S_{a}}{S_{a}-S_{b}}
$$

Computation of the ratio $\mathrm{R}$ at the edge location is not well defined, because the intensity of a blurred step-edge remains at half step-height at the center location for different scales and thus the denominator of the ratio $\left(S_{\mathrm{a}}-\mathrm{S}_{\mathrm{b}}\right)$ approaches zero. So, this ratio is unstable at the edge center and has thus a large stochastic error when it is computed close to the edge location. Computation at another location results in a systematic error.

\section{FIRST-ORDER DERIVATIVE-BASED METHODS}

We aim for low-cost accurate and precise local blur estimation based on the first-order derivative.

Therefore, we will exclude the following.

- To obtain accurate and precise results, we will exclude higher-order approaches because they are more expensive to compute and because the signal-to-noise ratio decreases for them. Recent results [12] already showed better performance based on a lower-order method.

- To obtain low-cost results, we will exclude methods that require a minimization or fitting procedure because it is computationally inefficient. This will enable a fast (parallel) implementation (e.g., on graphical processing units GPU's).

We will include the approach based on the gradient magnitude and step-height (similar to Cao [5]): 


$$
\sigma_{o}=\sqrt{\left(\frac{H}{L_{w} \sqrt{2 \pi}}\right)^{2}-\sigma_{a}^{2}}
$$

And we propose the following novel approach based on the gradient magnitude at two different scales:

$$
\sigma_{o}=\sqrt{\frac{\sigma_{b}^{2}-P \sigma_{a}^{2}}{P-1}} ; P=\left(\frac{L_{w, a}}{L_{w, b}}\right)^{2}
$$

where $\mathrm{L}_{\mathrm{w}, \mathrm{a}}$ and $\mathrm{L}_{\mathrm{w}, \mathrm{b}}$ are the gradient magnitudes at scale $\sigma_{\mathrm{a}}$ and $\sigma_{\mathrm{b}}$ respectively.

Eq. 11 was obtained by solving the following well-known equations for the gradient magnitude at a blurred step-edge at $\mathrm{x}=0$ for $\sigma_{\mathrm{o}}$ :

$$
L_{w, a}=\frac{H}{\sigma_{t a} \sqrt{2 \pi}} \exp \left(-\frac{x^{2}}{2 \sigma_{t a}^{2}}\right) ; L_{w, b}=\frac{H}{\sigma_{t b} \sqrt{2 \pi}} \exp \left(-\frac{x^{2}}{2 \sigma_{t b}^{2}}\right)
$$

Furthermore, we extended this approach by using only information up to the first-order derivatives, to handle small dislocations and to avoid root-finding or interpolation at sub-voxel locations.

$$
\sigma_{o}=\sqrt{\frac{\sigma_{b}^{2}-P * \sigma_{a}^{2}}{P-1}} ; P=\left(\frac{L_{w, a^{*}} \exp \left(-\left[\operatorname{erf}^{-1}\left(2 \frac{S_{b}-U_{1}}{H}-1\right)\right]^{2}\right)}{L_{w, b} * \exp \left(-\left[\operatorname{erf}^{-1}\left(2 \frac{S_{a}-U_{1}}{H}-1\right)\right]^{2}\right)}\right)^{2}
$$

The extension in Eq. 13 was obtained by solving the following equation of the blurred step edge for the complete term ($\left.\mathrm{x}^{2} /\left(2 \sigma_{\mathrm{ta}}{ }^{2}\right)\right)$, which is inside the exponent of Eq. 12 .

$$
S_{a}=U_{1}+\frac{U_{2}}{2}\left(1+\operatorname{erf}\left(\frac{x}{\sigma_{t a} \sqrt{2}}\right)\right) ; \leftrightarrow-\frac{x^{2}}{2 \sigma_{t a}^{2}}=-\left[\operatorname{erf}^{-1}\left(2 \frac{S_{b}-U_{1}}{H}-1\right)\right]^{2}
$$

For the term $\left(-\mathrm{x}^{2} /\left(2 \sigma_{\mathrm{tb}}{ }^{2}\right)\right)$, the same computation is made and both terms are substituted in Eq. 12 to derive Eq. 13. This allows us to avoid using the assumption that $\mathrm{x}=0$.

We will compare the two first-order derivative approaches of Eq. 11 and 13 to the method of Hu and Haan (Eq. 9) and the method of Cao (Eq. 10).

\section{EXPERIMENT AND RESULTS}

\subsection{Synthetic image data}

To quantitatively analyze the performance of the methods we used a one-dimensional blurred step edge $\left(\sigma_{0}\right)$ with white Gaussian noise $\left(\sigma_{i}=0.001\right)$. Of course, the methods can also be applied to multi-dimensional images, which will make them even more robust to the effects of noise [2]. The methods were applied without dislocation $(\mathrm{dx}=0)$ and with dislocation $(\mathrm{dx}>0)$. The step-height for Eq. 10 and Eq. 13 was determined as an average of the 7 pixels near $3 \sigma_{\mathrm{a}}$. This implementation was chosen to perform blur estimation in a local neighborhood, as with the other methods. The parameters for the five experiments (A-E) are described in Table 1.

Table 1. Parameters of the five synthetic experiments (A-E).

\begin{tabular}{|l|l|l|l|l|}
\hline \multicolumn{1}{|c|}{ Experiment } & \multicolumn{1}{|c|}{$\boldsymbol{\sigma}_{\mathbf{o}}$} & \multicolumn{1}{c|}{$\boldsymbol{\sigma}_{\mathbf{a}}$} & \multicolumn{1}{c|}{$\boldsymbol{\sigma}_{\mathbf{b}}$} & \multicolumn{1}{c|}{$\mathbf{d x}$} \\
\hline $\mathrm{A}$ & $0.7-7.4$ & 3.0 & $1.4 * \sigma_{\mathrm{a}}$ & 0.0 \\
\hline $\mathrm{B}$ & $0.7-7.4$ & 3.0 & $1.4 * \sigma_{\mathrm{a}}$ & 0.5 \\
\hline $\mathrm{C}$ & 2.0 & $3.0-31.8$ & $1.4 * \sigma_{\mathrm{a}}$ & 0.0 \\
\hline $\mathrm{D}$ & 2.0 & 3.0 & $1.4 * \sigma_{\mathrm{a}}$ & $0.004-0.5$ \\
\hline $\mathrm{E}$ & 0.7 & 3.0 & $1.4 * \sigma_{\mathrm{a}}$ & $0.004-0.5$ \\
\hline
\end{tabular}

Results of the synthetic experiment are shown in Table 2. Each row in this table shows a different experiment (A-E) and each column shows a different method (Eq. 9, 10, 11 and 13). Each method gives an estimation $\left(\sigma_{\text {est }}\right)$ of the original blur. On the vertical axis of each figure the relative error is shown $\left(\varepsilon=\left(\sigma_{\text {est }}-\sigma_{0}\right) / \sigma_{0}\right)$. On the horizontal axis, the varying parameter is shown, which is for the five experiments (A-E): $\sigma_{\mathrm{o}}, \sigma_{\mathrm{o}}, \sigma_{\mathrm{a}}, \mathrm{dx}$ and $\mathrm{dx}$ respectively. 
As expected, all methods - except our novel Eq. 13 - show a systematic error when they are applied at another location than the center of the edge $(\mathrm{dx}>0.1)$, especially when the original blur is small $\left(\sigma_{\mathrm{o}}<1.5\right)$. This systematic error hampers efficient implementation with a computation at pixel locations.

The results show that the method of $\mathrm{Hu}$ and Haan (Eq. 9) often has a large stochastic error when the dislocation is small. For this method, there is always a trade-off between systematic and stochastic errors (see Experiment 'E'). It only gives acceptable results when three conditions are met: $\sigma_{\mathrm{o}}<<\sigma_{\mathrm{a}}$ (because they use an approximation) and dx $>0.05$ (to avoid the stochastic error) and $\sigma_{0}>2$ (to avoid errors due to dislocation). The first condition hampers a local application, because large re-blurring kernels $\sigma_{\mathrm{a}}$ are more likely to interfere with neighboring structures. And the third condition is also problematic since the standard deviation of the PSF is often smaller than 2.

The approaches based on the first-order derivative (Eq. 10, 11 and 13) give good results. The approach based on Cao (Eq. 10) deteriorates when $\left(\sigma_{\mathrm{a}}<\sigma_{\mathrm{o}}\right)$. Furthermore, we observe in Experiment ' $\mathrm{C}$ ' that this method also deteriorates when $\left(\sigma_{\mathrm{o}}<<\sigma_{\mathrm{a}}\right)$.

The best performance is given by the approach based on Eq. 13, which corrects for small dislocations.

Table 2. Results of the five synthetic experiments (A-E) for four methods (Eq. 9, 10,11, 13). The vertical axis shows the relative error $\left(\varepsilon=\left(\sigma_{\text {est }}-\sigma_{\mathrm{o}}\right) / \sigma_{\mathrm{o}}\right)$ and the horizontal axis shows the varying parameter of the experiment.

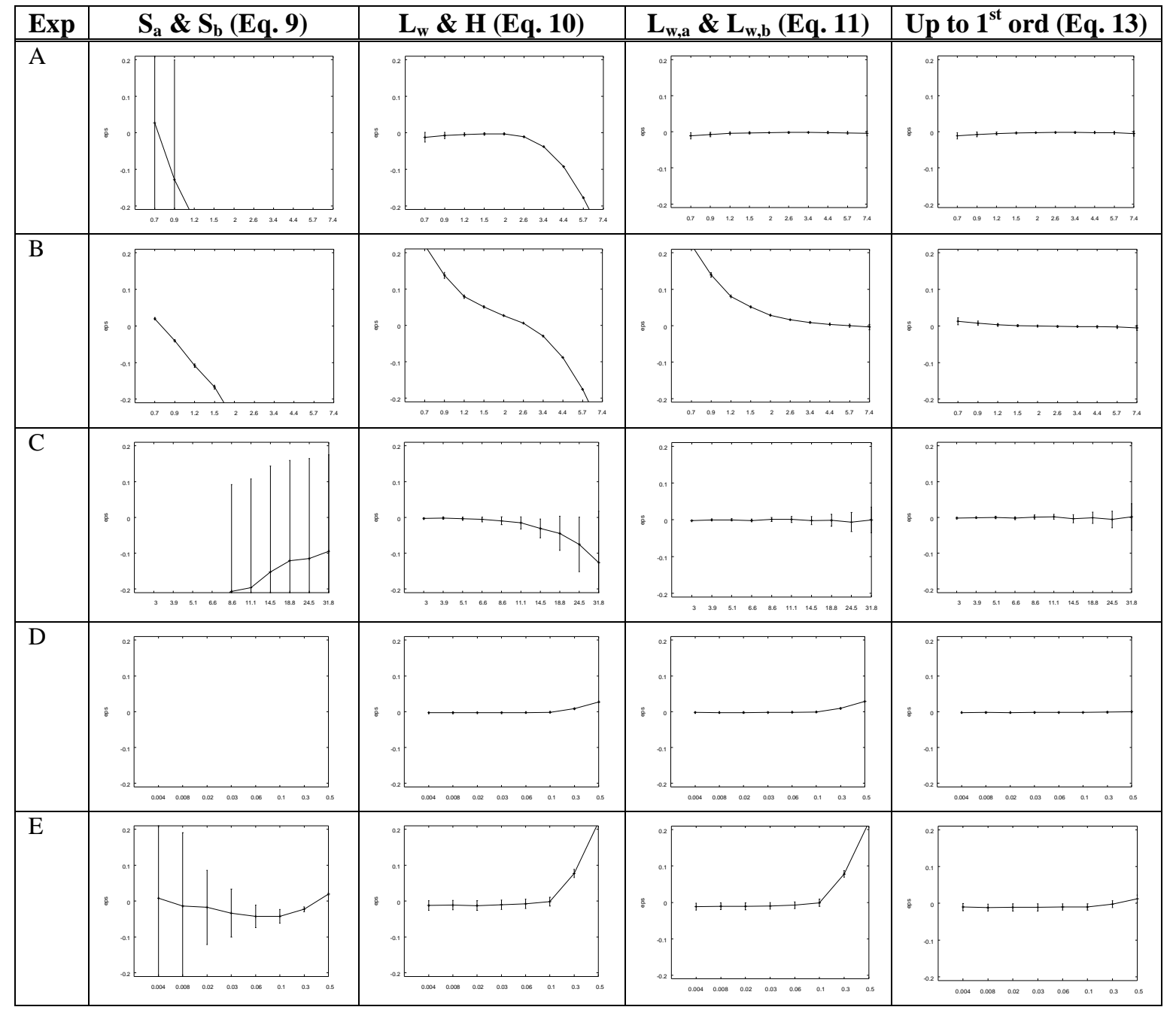




\subsection{Real image data}

We applied the blur estimation methods (Eq. 9, 10,11 and 13) to the 'cameraman' image at different levels of added blur $\left(\sigma_{\Delta}=0.0-7.0\right)$. The main difference between this image and synthetic data is that it contains not only one straight step edges but also curved edges and neighboring structures. The edge pixels are selected by using the zero crossings of the second-order derivative $\mathrm{L}_{\mathrm{ww}}$ and a minimal gradient magnitude $\mathrm{L}_{\mathrm{w}}$. The edge pixels are dilated to select a narrow region near the edge and the blur estimates are further dilated to improve the visibility in the figures of this paper. The methods were applied with $\sigma_{\mathrm{a}}=2.0$ and $\sigma_{\mathrm{b}}=2.8$. Figure 1 shows an example of the four different methods at $\left(\sigma_{\Delta}=4.0\right)$. Note the stability of the blur estimators at non-straight regions, such as the elbow.

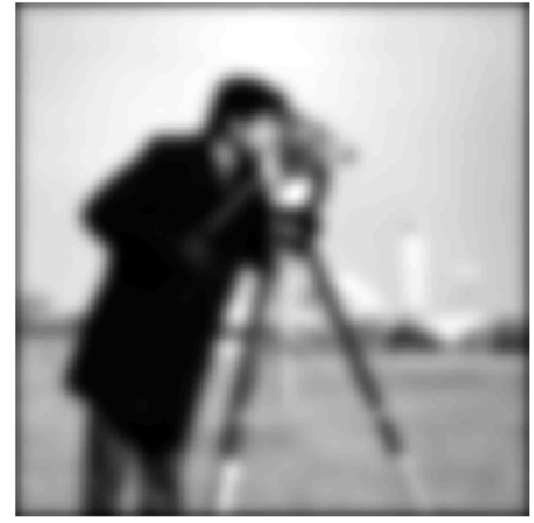

(a)

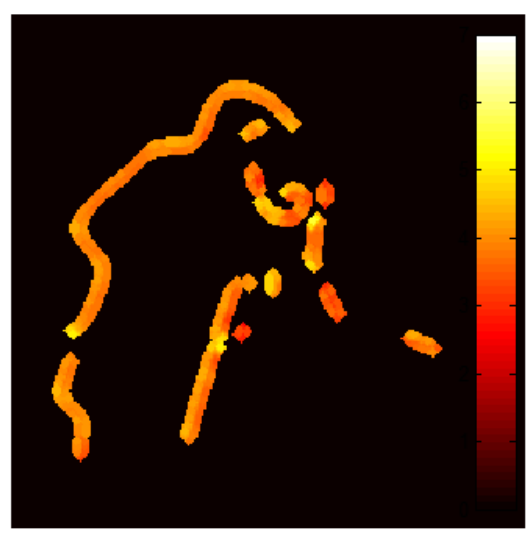

(d)

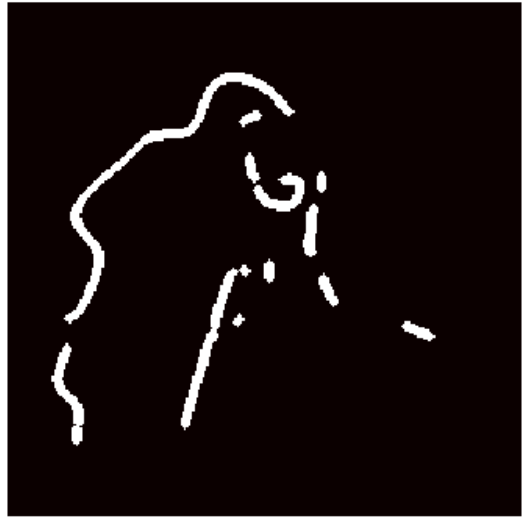

(b)

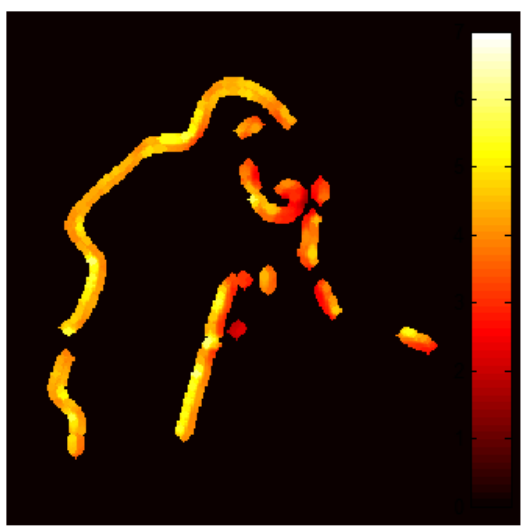

(e)

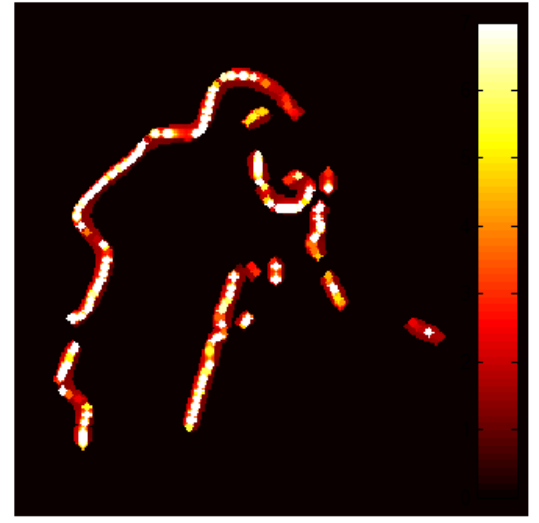

(c)

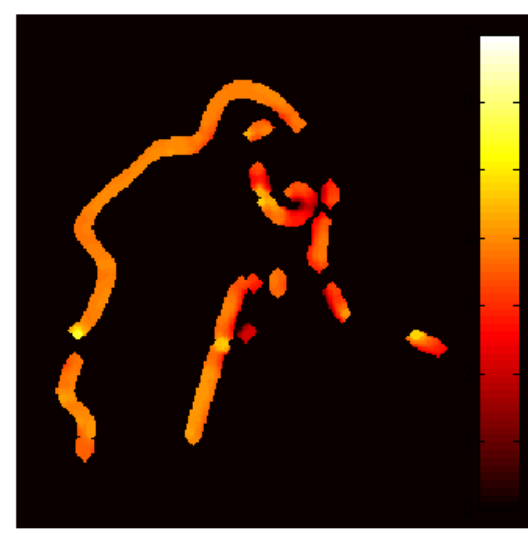

(f)

Figure 1. Blur estimation methods applied to the cameraman image with added blur $\sigma_{\Delta}=4.0$. Blurred input image (a), selected edge pixels (b), blur estimation method Eq. 9 (c), Eq. 10 (d), Eq. 11 (e) and Eq. 13 (f). Range of the color bar is [0 -7], where 4 is the level of applied blur. Note the very unstable estimation of Eq. 9 (c), the small variation due to dislocation and corners of Eq. 11 (e) and the stable and accurate estimation of the Eq. 13 (f).

Table 3 and Figure 2 show the average and standard deviations of the estimated blur in the cameraman image at different levels of additional blur. This figure shows that Eq. 9 is completely unstable and it shows that Eq. 11 and 13 are more accurate than Eq. 10.

In Table 3, the spread of Eq. (11) and Eq. (13) seems not to be different at $\sigma_{\Delta}=4.0$, although Figure 2e and 2f show a clear difference in stability. This is caused by the mixture of blur values on the left and right side of the image. When these estimates are separated it shows on the left side $4.5 \pm 0.5$ and $3.9 \pm 0.2$ for Eq. (11) and (13) respectively, and on the right side $3.9 \pm 0.8$ and $3.4 \pm 0.7$ for these equations. This clearly shows a much more stable estimate of Eq. (13) at places where a step edge is present. 
Table 3. Average and standard deviation of the estimated blur in the cameraman image at different levels of additional blur $\left(\sigma_{\Delta}\right)$.

\begin{tabular}{|l||c|c|c|c|c|c|c|c|}
\hline Method & $\boldsymbol{\sigma}_{\Delta}=\mathbf{0}$ & $\boldsymbol{\sigma}_{\Delta}=\mathbf{1}$ & $\boldsymbol{\sigma}_{\Delta}=\mathbf{2}$ & $\boldsymbol{\sigma}_{\Delta}=\mathbf{3}$ & $\boldsymbol{\sigma}_{\Delta}=\mathbf{4}$ & \multicolumn{1}{|c|}{$\boldsymbol{\sigma}_{\Delta}=\mathbf{5}$} & $\boldsymbol{\sigma}_{\Delta}=\mathbf{6}$ & $\boldsymbol{\sigma}_{\Delta}=\mathbf{7}$ \\
\hline Eq. (9) & $5.2 \pm 82$ & $4.0 \pm 20$ & $4.5 \pm 17$ & $6.6 \pm 29$ & $23.4 \pm 139$ & $23.3 \pm 106$ & $337 \pm 3319$ & $28.1 \pm 124$ \\
\hline Eq. (10) & $1.4 \pm 0.8$ & $2.0 \pm 0.8$ & $2.5 \pm 0.6$ & $3.3 \pm 0.5$ & $3.9 \pm 0.4$ & $4.4 \pm 0.3$ & $4.7 \pm 0.2$ & $5.0 \pm 0.2$ \\
\hline Eq. (11) & $1.3 \pm 2.1$ & $1.7 \pm 1.2$ & $2.0 \pm 0.8$ & $3.0 \pm 0.7$ & $4.1 \pm 0.8$ & $5.2 \pm 0.8$ & $6.0 \pm 0.6$ & $7.2 \pm 0.7$ \\
\hline Eq. (13) & $1.1 \pm 0.7$ & $1.3 \pm 1.0$ & $1.7 \pm 0.6$ & $2.6 \pm 0.6$ & $3.6 \pm 0.6$ & $4.6 \pm 0.5$ & $5.5 \pm 0.5$ & $6.6 \pm 0.4$ \\
\hline
\end{tabular}

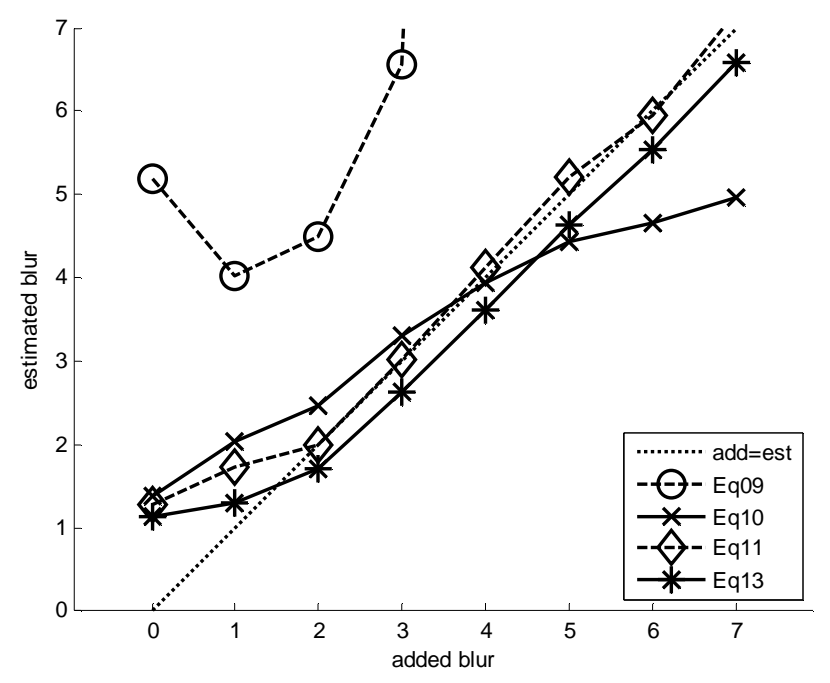

Figure 2. Average estimated blur (vertical axis) at different levels of added blur (horizontal axis).

Results on real data confirm results on synthetic data that Eq. 9 is completely unstable. The example in Figure 1 showed that Eq. 11 is sensitive to dislocations and corners and the quantitative results in Figure 2 showed that Eq. 10 is hampered by a systematic error. The results on synthetic and real data show that Eq. 13 gives the best blur estimate, but Eq. 11 also gives acceptable results close to the edge location and it is even easier to compute.

\section{CONCLUSIONS AND FUTURE WORK}

Blur estimation is an important technique for many practical applications, such as super resolution, image restoration, turbulence mitigation, deblurring and autofocus. Many techniques have been proposed to perform blur estimation in images and video. In this paper, we presented an efficient, accurate and precise blur estimator that can be computed at the edge location. Furthermore, it is robust against small variations due to dislocation or noise. The robustness against dislocation allows an efficient implementation based on computation at pixel locations in the neighborhood of an edge. The novel estimator is based on the first-order derivative. Our method is compared and benchmarked against previous state-of-the-art methods ([5][12]) under different levels of blur, dislocations and noise to gain insight in the robustness. The capabilities and limitations of each of the methods were analyzed and described. The experimental results show that the presented method is fast, unbiased and with low stochastic error. 


\section{REFERENCES}

[1] Bae, S., Durand, F., "Defocus magnification", Eurographics 26(3), (2007).

[2] Bouma, H., Vilanova, A., Vliet, L.J. van, Gerritsen, F.A., "Correction for the dislocation of curved surfaces caused by the PSF in 2D and 3D CT images", IEEE Trans. Pattern Analysis and Machine Intelligence 27(9), 1501-1507 (2005).

[3] Bouma, H., Olivan-Bescos, J., Vilanova, A., Gerritsen, F.A., "Unbiased vessel-diameter quantification based on the FWHM criterion", Proc. SPIE 6521, (2007).

[4] Bouma, H., Vilanova, A., Olivan-Bescos, J., Haar-Romeny, B.M. ter, Gerritsen, F.A., "Fast and accurate Gaussian derivatives based on B-splines", Scale Space LNCS 4485, (2007).

[5] Cao, G., Ni, R., "Edge-based blur metric for tamper detection", J. Information hiding and multimedia signal processing 1(1), 20-27 (2010).

[6] Chiang, M.-C., Boult, T.E., "Local blur estimation and super resolution”, IEEE CVPR, (1997).

[7] Dijk, J., Ginkel, M. van, Asselt, R.J. van, Vliet, L.J. van, Verbeek, P.W., “A new sharpness measure based on Gaussian lines and edges”, Computer Analysis of Images and Patterns LNCS 2756, 149-156 (2003).

[8] Eekeren, A. van, Schutte, K., Dijk, J., Schwering, P., "Turbulence compensation: an overview”, Proc. SPIE 8353, (2012).

[9] Eekeren, A. van, Kruithof, M., Schutte, K., Dijk, J., Iersel, M., Schwering, P., "Patch-based local turbulence compensation in anisoplanatic conditions", Proc. SPIE 8353, (2012).

[10] Eekeren, A. van, Schutte, K., Dijk, J., Lange, D.J.J. de, Vliet, L.J. van, "Super-resolution on moving objects and background", IEEE Int. Conf. Image Processing, 2709-2712 (2006).

[11]Elder, J.H., Zucker, S.W., "Local scale control for edge detection and blur estimation", IEEE Trans. Pattern Analysis and Machine Intelligence 20(7), 699-716 (1998).

[12] Hu, H. Haan, G. de., "Low cost robust blur estimation", Proc. IEEE Int. Conf. Image Processing, 617-620 (2006).

[13] Hu, H., Haan, G. de, "Adaptive image restoration based on local robust blur estimation", Proc. Advanced Concepts for Intelligent Vision Systems LNCS, 461-472 (2007).

[14] Kayargadde, V., [Feature extraction for image quality prediction], PhD thesis Technical University Eindhoven, The Netherlands, (1995).

[15] Pham, T.Q., [Spatiotonal adaptivity in super-resolution of under-sampled image sequences], PhD thesis Delft University of Technology, The Netherlands, (2006).

[16] Rooms, F., Ronsse, M., Pizurica, A., Philips, W., "PSF estimation with applications in autofocus and image restoration", Proc. IEEE Benelux Signal Processing Symposium, (2002).

[17]Zhang, W., Bergholm, F., "Multi-scale blur estimation and edge type classification for scene analysis", Int. J. Computer Vision 24(3), 219-250 (1997). 